\title{
ChemComm
}

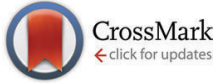

Cite this: Chem. Commun., 2017, 53,324

Received 2nd September 2016, Accepted 7th December 2016

DOI: $10.1039 / c 6 c c 07190 b$

\section{Monodentate coordination of the normally chelating chiral diamine $(R, R)-\mathrm{TMCDA} \dagger$}

\author{
Ana I. Ojeda-Amador, Antonio J. Martínez-Martínez, Alan. R. Kennedy, \\ David R. Armstrong and Charles T. O'Hara*
}

www.rsc.org/chemcomm

\begin{abstract}
After isolating an unusual binuclear, but monosolvated NaHMDS complex $\left[\{(R, R)-\mathrm{TMCDA}\} \cdot(\mathrm{NaHMDS})_{2}\right]_{\infty}$ which polymerises via intermolecular electrostatic $\mathrm{Na} \cdots \mathrm{Me}_{\mathrm{HMDs}}$ interactions, further $(R, R)$-TMCDA was added to produce the discrete binuclear amide $\left[\left\{\kappa^{2}-(R, R)-T M C D A\right\}\right.$. (NaHMDS) $)_{2}\left\{\kappa^{1}-(R, R)-\mathrm{TMCDA}\right\}$, whose salient feature is the unique monodentate coordination of one of the chiral diamine ligands.
\end{abstract}

Chiral diamine ligands, for example (-)-sparteine, its (+)-sparteine surrogate and $N, N, N^{\prime}, N^{\prime}$-(1R,2R)-tetramethylcyclohexane-1,2-diamine $[(R, R)$-TMCDA $]$ have attracted considerable attention in asymmetric synthesis in a whole host of transition metal catalysed methodologies. ${ }^{1}$ From an s-block perspective, when paired with an organolithium reagent it can be envisaged that 'chiral carbanions' are created, which can be used in subsequent enantioselective syntheses. ${ }^{2}$ Focusing particularly on the $C_{2}$-symmetric ligand $(R, R)$-TMCDA, it has come to prominence recently as the availability of the historically more widely utilised diamine (-)-sparteine, has been unreliable over the past few years. ${ }^{3}$ In terms of its coordination chemistry, $(R, R)$-TMCDA has worldwide interest and has been well studied. Over 50 metal complexes containing its ligated form have been reported, spanning both the $\mathrm{s}^{-}\left(\mathrm{Li},{ }^{4} \mathrm{Na},{ }^{4 e}\right.$ $\mathrm{K},{ }^{4 e}$ and $\left.\mathrm{Mg},{ }^{5}\right)$ and d-block metals $\left(\mathrm{Cu},{ }^{6} \mathrm{Zn},{ }^{7} \mathrm{Ru},{ }^{8} \mathrm{Pd},{ }^{9} \mathrm{Pt}^{10}\right.$ and $\left.\mathrm{Hg}^{11}\right)$. Within s-block chemistry and germane to this work, Strohmann has comprehensively studied $(R, R)$-TMCDA complexes of synthetically important organolithium reagents (such as ${ }^{t} \mathrm{BuLi}^{4},{ }^{4 a} \mathrm{MeLi},{ }^{4 b}$ ${ }^{\mathrm{i}} \mathrm{PrLi},{ }^{4 b}{ }^{s} \mathrm{BuLi},{ }^{4 b}{ }^{n} \mathrm{BuLi},{ }^{4 c} \mathrm{BH}_{3} \mathrm{P}(\mathrm{Ph})(\mathrm{Me}) \mathrm{CH}_{2} \mathrm{Li}^{4 d}{ }^{4 d} \mathrm{MeLi},{ }^{4 g} \mathrm{PhLi},{ }^{4 h}$ (allyl) $\mathrm{Li}^{4 h}$ and (benzyl) $\mathrm{Li}^{4 i}$ derivatives). An all-encompassing feature of all known structures is that the chiral diamine ligand adopts exclusively a $\kappa^{2}$-bidentate chelating mode. Due to the less flexible, fixed bite angle in $(R, R)$-TMCDA, with respect to that of $N, N, N^{\prime}, N^{\prime}$ tetramethylethylenediamine (TMEDA), ${ }^{12}$ it is a stronger chelating ligand than the latter, ${ }^{13}$ with a recent study noting that it 'displays

WestCHEM, Department of Pure and Applied Chemistry, University of Strathclyde, 295 Cathedral Street, Glasgow, G1 1XL, UK. E-mail: charlie.ohara@strath.ac.uk

$\dagger$ Electronic supplementary information (ESI) available: General synthetic procedures, crystal structure determinations and NMR spectroscopic data. CCDC 1501992 and 1501993. For ESI and crystallographic data in CIF or other electronic format see DOI: 10.1039/c6cc07190b no tendency to bind as a monodentate ligand. ${ }^{, 14}$ This has been attributed to the $\kappa^{1}$ (or by implication $\eta^{1}$ ) form of $(R, R)$-TMCDA inducing severe steric strain due to the juxtaposition of the metal$\mathrm{NMe}_{2}$ with the uncoordinated $\mathrm{NMe}_{2}$ group. The structural chemistry of alkali metal amide complexes continues to be an important topic of research. ${ }^{15}$ We have recently discovered that lithium and sodium 1,1,1,3,3,3-hexamethyldisilazide (LiHMDS and NaHMDS) can capture alkali metal halide salts in the presence of donor ligands to form ion pair metal anionic crown (MAC) complexes, for example $\left[\mathrm{Li}\{(R, R)-\mathrm{TMCDA}\}_{2}\right]^{+}\left[\mathrm{Li}_{5} \mathrm{HMDS}_{5} \mathrm{Cl}\right]^{-} \cdot{ }^{4 f, 16}$ A key starting material which remained hitherto elusive in our studies involving sodium is the $(R, R)$-TMCDA-solvated NaHMDS complex. Crystallisation of other donor ligated [e.g., $\mathrm{Me}_{6} \mathrm{TREN}^{17}$ and (-)-sparteine $\left.{ }^{18}\right]$ NaHMDS complexes has proven difficult, although the polymeric TMEDA $\left[(\mu \text {-TMEDA }) \cdot(\mathrm{NaHMDS})_{2}\right]_{\infty}{ }^{19}$ and $N, N, N^{\prime}, N^{\prime}$-tetramethylpropanediamine (TMPDA) [( $\mu$-TMPDA). $\left.(\mathrm{NaHMDS})_{2}\right]_{\infty}{ }^{20}$ complexes, which propagate via the nonchelating diamine ligand, are known (Fig. 1). These have similar structural motifs to Williard's lithium diisopropylamide (LDA) complex $\left[\left(\mu \text {-TMEDA) } \cdot(\mathrm{LDA})_{2}\right]_{\infty} \cdot{ }^{19}\right.$

In an effort to prepare the $(R, R)$-TMCDA complex of NaHMDS, an equimolar mixture of NaHMDS and $(R, R)$-TMCDA was combined in $n$-hexane medium and left to stir at ambient temperature for 1 hour (Scheme 1). The reaction mixture was then cooled to $-33{ }^{\circ} \mathrm{C}$ and crystals suitable for X-ray crystallographic analysis deposited after 48 hours (27\% non-optimised, crystalline yield; maximum yield 50\% based on $(R, R)$-TMCDA consumption). X-ray data reveal the mono$(R, R)$-TMCDA, binuclear $\left[\{(R, R) \text {-TMCDA }\} \cdot(\mathrm{NaHMDS})_{2}\right]_{\infty} \mathbf{1}$ (Fig. 2a). There are six crystallographically distinct but essentially chemically

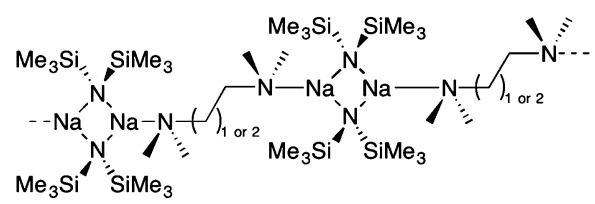

Fig. 1 Structures of previously known polymeric $\left[(\mu-\mathrm{TMEDA}) \cdot(\mathrm{NaHMDS})_{2}\right]_{\infty}$ and $\left[(\mu-\mathrm{TMPDA}) \cdot(\mathrm{NaHMDS})_{2}\right]_{\infty}$. 


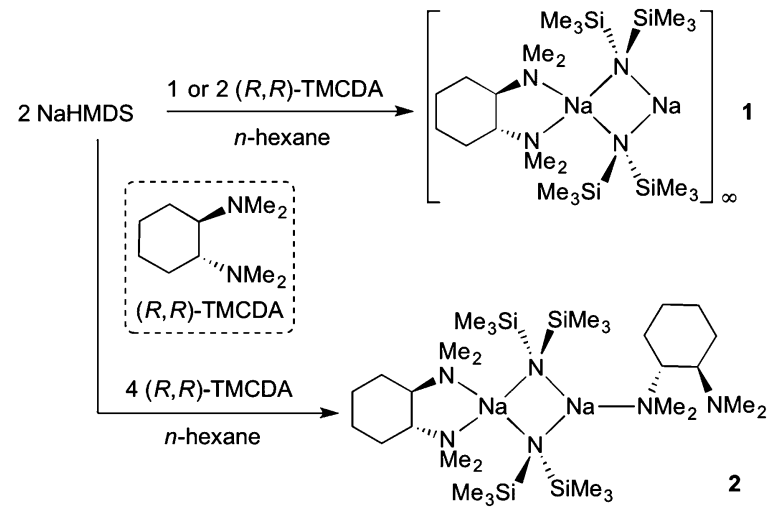

Scheme 1 Syntheses of $\left[\{(R, R)-\mathrm{TMCDA}\} \cdot(\mathrm{NaHMDS})_{2}\right]_{\infty} 1$ and $\left[\left\{\kappa^{2}-(R, R)-\right.\right.$ TMCDA $\left.\} \cdot(\mathrm{NaHMDS})_{2}\left\{\kappa^{1}-(R, R)-\mathrm{TMCDA}\right\}\right] 2$.

equivalent molecules of $\left[\{(R, R)-\mathrm{TMCDA}\} \cdot(\mathrm{NaHMDS})_{2}\right]$ in the structure of $\mathbf{1}$, thus for brevity only one is discussed here. Interestingly, the empirical formula of 1, i.e., [(donor).(NaHMDS $\left.)_{2}\right]$ is identical to that for the aforementioned TMEDA and TMPDA derivatives; however, in keeping with previously known $(R, R)$-TMCDA complexes, the diamine adopts a chelating bonding mode, and with respect to the $\mathrm{N}$ donor atoms, renders one $\mathrm{Na}$ metal centre (Na1) four-coordinate in a distorted tetrahedral arrangement (bond angles range from 68.70(9) to $151.55(10)^{\circ}$, see $\mathrm{ESI} \dagger$ for full details). Additionally, Na1 has two long $\mathrm{Na} \cdots$ Me interactions with a methyl group from each HMDS

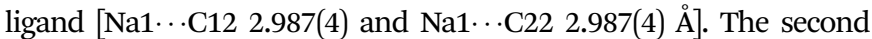
Na metal centre (Na2) remains only two-coordinate with respect to the bridging amido $\mathrm{N}$ atoms. To satisfy this electron deficiency, $\mathrm{Na} 2$

a)

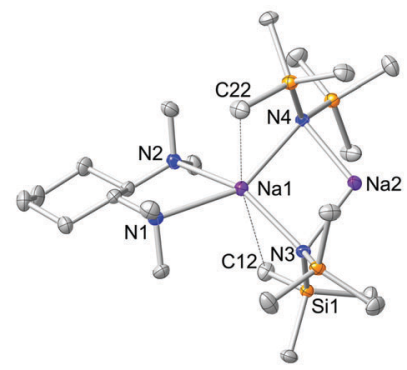

b)

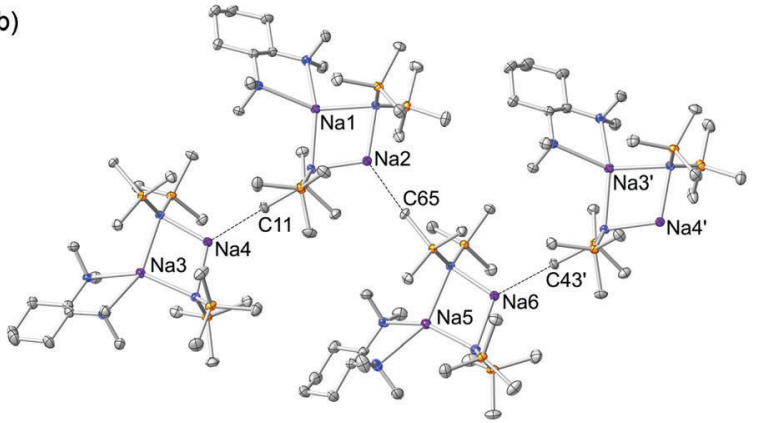

Fig. 2 (a) Molecular structure of $\left[\{(R, R)-\mathrm{TMCDA}\} \cdot(\mathrm{NaHMDS})_{2}\right]_{\infty} 1$ showing one molecule from the asymmetric unit. Hydrogen atoms omitted for simplicity and thermal ellipsoids are displayed at 35\% probability. (b) Section of the zigzag polymeric chain of 1 . The dashed lines illustrate $\mathrm{Na} \cdot \mathrm{Me}\left(\mathrm{SiMe}_{2}\right)$ interactions. The symmetry operation used to generate the atoms labelled with ' is $-x+1, y+1 / 2,-z+1$. engages a solitary intermolecular $\mathrm{Na} \cdots \mathrm{Me}\left(\mathrm{SiMe}_{2}\right)[\mathrm{Na} 2 \cdots \mathrm{C} 65$ distance, 2.818(4) $\AA$ ] electrostatic interaction (Fig. 2b), which is short in comparison to known literature examples [range $\left.\mathrm{Na} \cdots \mathrm{Me}\left(\mathrm{SiMe}_{2}\right) \quad 2.947-3.138 \AA\right]{ }^{21}$ This sole intermolecular $\mathrm{Na} \cdots \mathrm{Me}$ interaction induces propagation of binuclear units in a zigzag polymer chain. This change in the coordination chemistry of $(R, R)$-TMCDA in 1 with respect to the bridging TMEDA and TMPDA ligands in the aforementioned polymeric sodium amides emphasises the propensity for the chiral 1,2-diamine to remain as a chelating ligand rather than binding in a monodentate fashion. As a consequence of this coordination mismatch, significantly shorter $\mathrm{Na} 2-\mathrm{N}_{\mathrm{HMDS}}$ bonds (mean distance, $2.356 \AA$ ) are observed when compared with Na1- $\mathrm{N}_{\mathrm{HMDS}}$ bonds (mean distance, $2.530 \AA$ ). Despite utilising a 1:1 ratio of NaHMDS: $(R, R)$-TMCDA in this synthesis, it is clearly evident that the ultimate ratio in 1 is $2: 1$. When this optimised ratio is used in the synthesis, $\mathbf{1}$ was again the sole product isolated ( $36 \%$ crystalline yield).

Complex 1 is a rare example of a solvated sodium amide which contains an unsolvated Na site. Bochmann revealed the mono(tetrahydrofuran), mono(THF), complex [(THF).(NaHMDS $)_{2}$ ] where one $\mathrm{Na}$ atom is two coordinate whilst the other binds to the ether to render it three coordinate. ${ }^{22}$ Interestingly, seven years prior to this report Dehnicke published the bis(THF) analogue $\left[(\mathrm{THF})_{2} \cdot(\mathrm{NaHMDS})_{2}\right]$ where both $\mathrm{Na}$ atoms are three coordinate. ${ }^{23}$ This begged the question: 'could the coordinatively unsaturated (Lewis acidic) Na atom in 1, act as a host for another Lewis base?'

A logical route to address this question would be to utilise monodentate donors such as THF and diethylether, in an attempt to saturate the deficient metal centre; but, it is highly likely that these strong $\sigma$-donors would also displace the chelating $(R, R)$-TMCDA ligand. Therefore to maintain synthetic simplicity, we repeated the preparation of $\mathbf{1}$ but employing an excess (two molar equivalents) of $(R, R)$-TMCDA with respect to NaHMDS in an attempt to coordinate a second molecule of the Lewis base ligand to the donor-free metal centre. High quality crystals (39\% crystalline yield) were obtained by storing the resultant solution at $-33{ }^{\circ} \mathrm{C}$ for $24 \mathrm{~h}$, which were analysed by $\mathrm{X}$-ray crystallography and were pleasingly found to be the target bis(solvated) derivative $\left[\left\{\kappa^{2}-(R, R)-\mathrm{TMCDA}\right\} \cdot(\mathrm{NaHMDS})_{2}\left\{\kappa^{1}-(R, R)\right.\right.$ TMCDA $\}] 2$ (Fig. 3). The distorted tetrahedral coordination sphere of $\mathrm{Na1}$ in 2 (bond angles around Na1 range from 66.90(6) to 151.05(8), see ESI†) is essentially identical to that found in 1, exhibiting additional long contacts with a methyl group from each HMDS

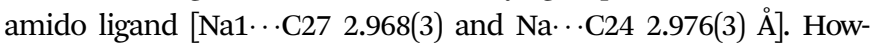
ever, the second sodium metal centre, $\mathrm{Na} 2$, is additionally coordinated to an extra molecule of $(R, R)$-TMCDA, giving rise to a distorted trigonal planar geometry. As such there are two distinct coordinated diamine ligands within the structure of 2 . Undoubtedly, the most eye-catching feature is that one $(R, R)$-TMCDA ligand adopts a previously unseen $\kappa^{1}$-coordination mode. To change from a $\kappa^{2}$ - to a $\kappa^{1}$-coordination mode, it appears that inversion of the $\mathrm{N} 1$ atom of the $(R, R)$-TMCDA has occurred, no longer allowing the ligand to chelate to Na2 (Fig. 3).

Complex 2 is a discrete dimeric entity, despite the potential availability for $\mathrm{N} 2$ to coordinate further. In theory, this could be 


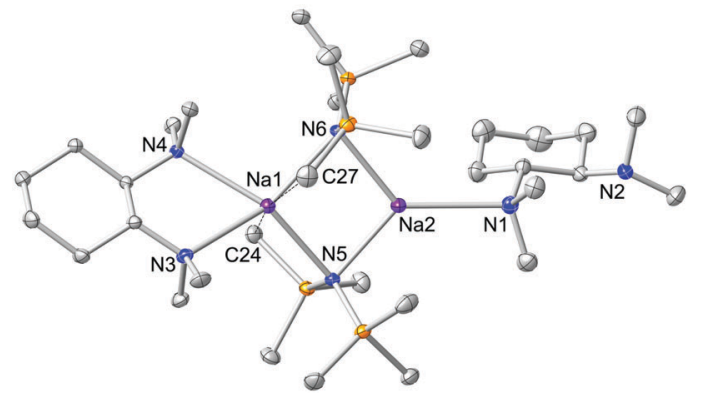

Fig. 3 Molecular structure of $\left[\left\{\kappa^{2}-(R, R)-\mathrm{TMCDA}\right\} \cdot(\mathrm{NaHMDS})_{2}\left\{\kappa^{1}-(R, R)-\right.\right.$ TMCDA\}] 2. Hydrogen atoms and one disordered component of the mono-dentate $(R, R)$-TMCDA ligand are omitted for simplicity. Thermal ellipsoids are displayed at $35 \%$ probability.

achieved if this $\mathrm{N}$ atom could also invert thus allowing an additional exo-coordination site; however, it is unlikely that this would occur due to high steric strain (buttressing). ${ }^{14}$ The $\kappa^{1}$-coordinated $(R, R)$-TMCDA is disordered over two domains, but its atomic connectivity and geometry are unequivocal. The $\kappa^{2}$ - and the hitherto unseen $\kappa^{1}$-coordination mode $(R, R)$ TMCDA observed in 2 can be compared with DFT calculations (at the B3P86/6-311+ $\mathrm{G}^{*}$ level) performed for its diamine relative (-)-sparteine (Fig. 4). ${ }^{24}$ It has been shown that when (-)-sparteine binds to a metal complex, it always adopts a chelating ' $c i s$ ' configuration. However, in the absence of a metal complex, it is actually slightly more stable (by $3.4 \mathrm{kcal} \mathrm{mol}^{-1}$ ) in a ring-flipped 'trans' configuration [akin to our $\kappa^{1}$-coordinated $(R, R)$-TMCDA] where the lone pairs of electron present on the $\mathrm{N}$ atoms are not adjacent to each other. We have performed similar DFT studies (ESI $\dagger)$ on $(R, R)$-TMCDA and have shown that there is negligible difference (less than $1 \mathrm{kcal} \mathrm{mol}^{-1}$ ) between the potentially $\kappa^{1}$ - and $\kappa^{2}$-coordination modes.

As $\mathbf{1}$ and $\mathbf{2}$ are both highly soluble in non-polar hydrocarbon and arene solutions, solutions of these compounds were studied by NMR spectroscopy. Using ${ }^{1} \mathrm{H}$ NMR spectroscopy, it was evident that the expected $1: 2$ and $2: 2(R, R)$-TMCDA:HMDS ratios were observed respectively. For $\mathbf{1}$, a single amido resonance (at $\delta 0.25$ ) was observed and the (R,R)-TMCDA resonances (at $\delta 2.01,1.90,1.47$ and 0.74$)$ in $\mathrm{C}_{6} \mathrm{D}_{6}$ solution appeared to correspond to a metallocoordinated ligand (see ESI $\dagger$ for full details). For 2, the amido resonance appears at $\delta 0.31$ in the same solvent. If the solid state structure of $\mathbf{2}$ was to be retained in solution, two unique sets of $(R, R)$-TMCDA resonances would be expected. In reality a single set of resonances (at $\delta 2.06,1.99,1.51$ and 0.80 in $\mathrm{C}_{6} \mathrm{D}_{6}$ solution) is observed. This indicates that a single $(R, R)$-TMCDA environment exists at $300 \mathrm{~K}$ in arene solution, indeed, a variable temperature
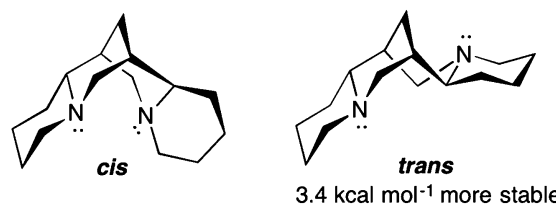

Fig. 4 Relative stabilities of cis and trans isomers of uncoordinated (-)-sparteine. ${ }^{24}$
NMR spectroscopic study of 2 in $\left[\mathrm{D}_{8}\right]$-toluene solution unveiled that this situation was maintained even at low temperature (down to $206 \mathrm{~K}$, see ESI $\dagger$ ). In addition, ${ }^{1} \mathrm{H}$ and ${ }^{13} \mathrm{C}$ NMR spectra obtained in non-polar $\left[\mathrm{D}_{12}\right]$-cyclohexane also reveal this situation (see ESI $\dagger$ ). Therefore due to the steric bulk of the HMDS ligands within the molecule [thus precluding a dual $\kappa^{2}$-situation for the $(R, R)$-TMCDA ligands], it is likely that the spectra show a time-averaged situation between dynamic $\kappa^{1}$ - and $\kappa^{2}$-coordinated $(R, R)$-TMCDA ligands.

In closing, we have shown that counter to previous studies, $(R, R)$-TMCDA can indeed bind to an alkali metal in a nonchelating $\kappa^{1}$-manner.

This work was supported by the EPSRC (through a Career Acceleration Fellowship to CTOH, EP/J001872/1 and EP/ L001497/1). The authors would like to thank Professors Mulvey and Hevia, and Dr Robertson for useful discussions. We would also like to thank Dr Claire McGoldrick for help with artwork design. The research data associated with this paper is openly available at http://dx.doi.org/10.15129/103ad89d-550d-44ad8aa8-62d54c47e3ac.

\section{Notes and references}

1 (a) K. Mikami and M. Yamanaka, Chem. Rev., 2003, 103, 3369; (b) R. Noyori, Adv. Synth. Catal., 2003, 345, 15; (c) V. Bette, A. Mortreux, F. Ferioli, G. Martelli, D. Savoia and J.-F. Carpentier, Eur. J. Org. Chem., 2004, 3040.

2 (a) D. Hoppe and T. Hense, Angew. Chem., Int. Ed. Engl., 1997, 2282; (b) J. Clayden, Organolithiums: Selectivity for Synthesis, Pergamon, New York, 2002; (c) O. Chuzel and O. Riant, Top. Organomet. Chem., 2005, 15, 59; (d) C.-A. B. Ferber, H. B. Kagan, O. Lafon and P. Lesot, Tetrahedron: Asymmetry, 2008, 19, 2666; (e) P. O'Brien, Chem. Commun., 2008, 655; $(f)$ Q. Perron, J. Praz and A. Alexakis, Tetrahedron: Asymmetry, 2009, 20, 1004; $(g)$ J. Praz, J. Graff, L. Egger, L. Guénée, S. Wagschal, E. P. Kündig and A. Alexakis, Chem. Commun., 2015, 51, 16912.

3 J. D. Firth, P. O'Brien and L. Ferris, Org. Biomol. Chem., 2014, $12,9357$.

4 (a) C. Strohmann and V. H. Gessner, Angew. Chem., Int. Ed., 2007, 46, 8281; (b) C. Strohmann and V. H. Gessner, J. Am. Chem. Soc., 2007, 129, 8952; (c) C. Strohmann and V. H. Gessner, J. Am. Chem. Soc., 2008, 130, 11719; (d) V. H. Gessner, S. Dilsky and C. Strohmann, Chem. Commun., 2010, 46, 4719; (e) P. García-Álvarez, A. R. Kennedy, C. T. O'Hara, K. Reilly and G. M. Robertson, Dalton Trans., 2011, 40, 5332; $(f)$ A. R. Kennedy, R. E. Mulvey, C. T. O'Hara, G. M. Robertson and S. D. Robertson, Angew. Chem., Int. Ed., 2011, 50, 8375; $(g)$ K. Götz, V. H. Gessner, C. Unkelbach, M. Kaupp and C. Strohmann, Z. Anorg. Allg. Chem., 2013, 639, 2077; (h) P. K. Eckert, B. Schnura and C. Strohmann, Chem. Commun., 2014, 46, 4719; (i) S. G. Koller, U. Kroesen and C. Strohmann, Chem. - Eur. J., 2015, 21, 641.

5 J. Francos, B. J. Fleming, P. García-Álvarez, A. R. Kennedy, K. Reilly, G. M. Robertson, S. D. Robertson and C. T. O'Hara, Dalton Trans., 2014, 43, 14424 .

6 (a) A. P. Cole, D. E. Root, P. Mukherjee, E. I. Solomon and T. D. P. Stack, Science, 1996, 273, 1848; (b) E. C. Brown, J. T. York, W. E. Antholine, E. Ruiz, S. Álvarez and W. B. Tolman, J. Am. Chem. Soc., 2005, 127, 13752; (c) A. P. Cole, V. Mahadevan, L. Mirica, X. Ottenwaelder and T. D. P. Stack, Inorg. Chem., 2005, 44, 7345; (d) J. T. York, I. Bar-Nahum and W. B. Tolman, Inorg. Chem., 2007, 46, 8105; (e) J. E. Bercaw, G. S. Chen, J. A. Labinger and B.-L. Lin, J. Am. Chem. Soc., 2008, 130, 17654; $(f)$ P. Verma, J. Weir, L. Mirica and T. D. P. Stack, Inorg. Chem., 2011, 50, 9816.

7 (a) H. Y. Lee, J. U. Yoon and J. H. Jeong, Acta Crystallogr., Sect. E: Struct. Rep. Online, 2007, 63, m2471; (b) P. K. Eckert, I. d. S. Vieira, V. H. Gessner, J. Borner, C. Strohmann and S. Herres-Pawlis, Polyhedron, 2013, 49, 151.

8 W.-C. Cheng, W.-Y. Yu, J. Zhu, K.-K. Cheung, S.-M. Peng, C.-K. Poon and C.-M. Che, Inorg. Chim. Acta, 1996, 242, 105. 
9 (a) S. V. Pavlova, Y.-S. Wen and S. I. Chan, Acta Crystallogr., Sect. E: Struct. Rep. Online, 2003, 59, m792; (b) G. Lu and H. C. Malinakova, J. Org. Chem., 2004, 69, 4701; (c) N. Miklasova, E. Fischer-Fodor, R. Miklas, L. Kuckova, J. Kozisek, T. Liptaj, O. Soritau, J. Valentova and F. Devinsky, Inorg. Chem. Commun., 2014, 46, 229.

10 M. Benedetti, G. Tamasi, R. Cini and G. Natile, Chem. - Eur. J., 2003, 9, 6122.

11 P. K. Eckert, V. H. Gessner, M. Knorr and C. Strohmann, Inorg. Chem., 2012, 51, 8516.

12 (a) D. Barr, W. Clegg, R. E. Mulvey, R. Snaith and D. S. Wright, J. Chem. Soc., Chem. Commun., 1987, 716; (b) M. Westerhausen, M. Wieneke, W. Ponikwar, H. Nöth and W. Schwarz, Organometallics, 1998, 17, 1438; (c) M. A. Beswick, C. N. Hamer, P. R. Raithby, A. Steiner, M. Tombil and D. S. Wright, J. Organomet. Chem., 1999, 573, 267; (d) E. Hevia, D. J. Gallagher, A. R. Kennedy, R. E. Mulvey, C. T. O'Hara and C. Talmard, Chem. Commun., 2004, 2422; (e) M. M. Meinholz and D. Stalke, Eur. J. Inorg. Chem., 2011, 4578.

13 (a) B. L. Lucht, M. P. Bernstein, J. F. Remenar and D. B. Collum, J. Am. Chem. Soc., 1996, 10707; (b) J. F. Remenar, B. L. Lucht and D. B. Collum, J. Am. Chem. Soc., 1997, 119, 5567; (c) D. Hoffmann and D. B. Collum, J. Am. Chem. Soc., 1998, 120, 5810; (d) J. L. Rutherford, D. Hoffmann and D. B. Collum, J. Am. Chem. Soc., 2002, 124, 264.

14 T. S. De Vries, A. M. Bruneau, L. R. Liou, H. Subramanian and D. B. Collum, J. Am. Chem. Soc., 2013, 135, 4103.

15 (a) R. Michel, T. Nack, R. Neufeld, J. M. Dieterich, R. A. Mata and D. Stalke, Angew. Chem., Int. Ed., 2013, 52, 734; (b) R. E. Mulvey and
S. D. Robertson, Angew. Chem., Int. Ed., 2013, 52, 11470-11487; (c) R. Neufeld, R. Michel, R. Herbst-Irmer, R. Schöne and D. Stalke, Chem. - Eur. J., 2016, 22, 12340-12346.

16 A. I. Ojeda-Amador, A. J. Martínez-Martínez, A. R. Kennedy and C. T. O'Hara, Inorg. Chem., 2015, 54, 9833.

17 D. M. Cousins, M. G. Davidson, C. J. Frankis, D. García-Vivo and M. F. Mahon, Dalton Trans., 2010, 39, 8278.

18 N. M. Clark, P. García-Álvarez, A. R. Kennedy, C. T. O'Hara and G. M. Robertson, Chem. Commun., 2009, 5835.

19 M. P. Bernstein, F. E. Romesberg, D. J. Fuller, A. T. Harrison, D. B. Collum, Q. Y. Liu and P. G. Williard, J. Am. Chem. Soc., 1992, 114, 5100.

20 K. W. Henderson, A. E. Dorigo, Q. Y. Liu and P. G. Williard, J. Am. Chem. Soc., 1997, 119, 11855.

21 A recent CCDC search (C. R. Groom, I. J. Bruno, M. P. Lightfoot and S. C. Ward, Acta Crystallogr., Sect. B: Struct. Sci., Cryst. Eng. Mater., 2016, $72,171)$ reveals a selection of examples comprising $\mathrm{Na} \cdots \mathrm{Me}(\mathrm{Si})$ interactions, for example see: F. Antolini, P. B. Hitchcock, M. F. Lappert and P. Merle, Chem. Commun., 2000, 1301; O. Bénaud, J.-C. Berthet, P. Thuéry and M. Ephritikhine, Inorg. Chem., 2010, 49, 8117.

22 Y. Sarazin, S. J. Coles, D. L. Hughes, M. B. Hursthouse and M. Bochmann, Eur. J. Inorg. Chem., 2006, 3211.

23 M. Karl, G. Seybert, W. Massa, K. Harms, S. Agarwal, R. Maleika, W. Stelter, A. Greiner, W. Heitz, B. Neümuller and K. Dehnicke, Z. Anorg. Allg. Chem., 1999, 625, 1301.

24 K. B. Wiberg and W. F. Bailey, J. Mol. Struct., 2000, 556, 239. 